\title{
Reformar ou não reformar? Análise da influência da forma na customização de projetos de apartamentos
}

\author{
- Cristiana Griz \\ Universidade Federal de Pernambuco, Brasil \\ crisgriz@gmail.com \\ - Vanessa Guedes \\ Universidade Federal de Pernambuco, Brasil \\ vanessa.aguedes17@gmail.com
}

To reform or not to reform? Analysis of the influence of the shape in the customization of apartment projects

\begin{abstract}
The starting point for this research was carried out through an investigation that measured in what extent original projects proposed by the real estate market were customized, resulting in Reformed Projects. It is known that many factors can influence this customization process. This phase of the research is restricted to formal and constructive factors, aiming to analyze how these can influence the customization. The shape grammar, used as an analytical tool, showed that the shape of the original projects had little influence on the result of the reformed ones, indicating that factors of other nature may be more decisive in the customization process.
\end{abstract}

Keywords: Shape Grammar, Apartment Projectsan

\section{Introdução}

Esse artigo apresenta parte dos resultados de uma pesquisa intitulada Projetos de apartamentos do Recife: caracterização dos modos de morar do século XXI (Griz, 2014). O objetivo geral é realizar um diagnóstico sobre os projetos de apartamentos de alto e médio padrão produzidos pelo mercado imobiliário do Recife, para poder propor estratégias capazes de guiar a concepção de tais projetos, de maneira que seus atributos construtivos e morfológicos (formais e configuracionais) facilitem, em certa medida, as reformas. Aqui, são apresentados os resultados parciais da análise dos atributos formais dos projetos.

Uma das principais funções da habitação, seja ela casa unifamiliar ou apartamento, é oferecer as condições para que determinadapessoa(ougrupodelas-afamília)desempenhecertas atividades domésticas de acordo com seus modos de habitar. Esse último, por sua vez, é alterado conjuntamente com a estrutura de valores de uma determinada sociedade. É compreensível, portanto, que à medida que essa estrutura de valores se altera, mudam-se também os elementos programáticos da habitação. Essa é uma das principais razões para a existência de reformas e adaptações em uma habitação (Hanson, 1998; Trigueiro, 1994). Em algumas cidades brasileiras (Griz, 2012, França, 2008; Paula, 2007) é comum adquirir um apartamento - produto caracterizado pela homogeneização de soluções projetuais (Tramontano, 2004; Brandão, 2002) e customiza-lo1.

1 Termo aplicado com o sentido de adaptar, personalizar um produto às preferências do cliente (customer).
O ponto de partida para esta investigação se deu a partir de uma pesquisa (Griz, 2012) que mensurou (através do Grau de Alteração do Projeto - GAP) o quanto projetos de apartamentos propostos pelo mercado imobiliário (os Projetos Originais POs) eram customizados por seus compradores, resultando nos Projetos Reformados (PRs). No contexto recifense, foi visto que essas customizações ressaltam certa incompatibilidade entre o projeto ofertado e determinadas demandas para abrigar o habitar contemporâneo (Griz, Amorim \& Loureiro, 2014). Além disso, podem gerar atraso e prejuízo financeiro para os construtores (que, consequentemente, repassam esses custos para os clientes).

Esses dados ressaltam a necessidade de minimizar: (1) essa incompatibilidade; (2) os custos do inevitável processo de customização. $\mathrm{Na}$ tentativa de superar esses inconvenientes, duas estratégias são lançadas: (1) a customização em massa de projetos de apartamentos, para garantir que o projeto seja adequado às necessidades dos clientes; (2) o desenvolvimento de projetos originais de apartamentos com um alto grau de flexibilidade para facilitar o inevitável processo de customização.

A customização em massa aplicada a projetos de apartamentos é uma estratégia que além de garantir que o apartamento ofereça um alto grau de satisfação aos moradores $^{2}$, pode ser tão econômica quanto à produção em massa. Segundo Kolarevic (2001, p. 122-123, tradução nossa),

2 Segundo Duarte (2007), um elevado nível de customização do projeto conduz a uma elevada satisfação do usuário. 
A noção de singularidade é agora tão econômica e fácil de alcançar quanto a repetição [...] agora nos esforçamos para customização em massa, trazendo os benefícios da produção da fábrica para a criação de um componente único ou de uma série de elementos semelhantes diferenciadas através da variação controlado digitalmente.

E são justamente essas tecnologias digitais, já consolidadas nas indústrias automotiva, aeronáutica e naval, que estão transformando os processos de projeto e a prática arquitetônica. Fischer e Herr (2001) reforçam esse argumento mostrando a relevância do uso de sistemas generativos ${ }^{3}$ no processo de projeto e na produção arquitetônica, como a gramática da forma (Stiny \& Gips, 1972), por exemplo. Esta vem sendo utilizada para a geração sistemática de soluções projetuais customizadas, inclusive em projetos de habitação em resposta à padronização das plantas (Duarte, 2005; Mussi, 2011; Eloy, 2012).

Nesse sentido, as duas estratégias propostas - tanto a customização de projetos de apartamentos, quanto projetos originais com algo potencial para ser customizados, podem ser alcançados através da aplicação do formalismo da gramática da forma e do uso de modelagem paramétrica para sua implementação. A primeira, através da criação de uma gramática paramétrica da customização; a segunda, da criação de uma gramática original paramétrica, codificada por um programa de computador, que gere projetos de apartamentos que apresentem um alto potencial de customização.

O desenvolvimento de gramáticas com tal complexidade é um processo longo, que demanda conhecimento e o uso de diversas tecnologias digitais. A modelagem paramétrica, por exemplo, é utilizada com o intuito de otimizar o processo de geração de diferentes soluções, bem como de permitir a definiçãodediferentesformatosdecômodos, a partir dainserção de parâmetros, como por exemplo, quantidade de vértices e dimensão dos lados. No entanto, tendo em vista esse longo caminho que precisa ser percorrido para que uma gramática seja implementada, esta etapa se dedica aos primeiros passos do seu desenvolvimento, pensando em soluções que, embora sejam preliminares, já vislumbram um desenvolvimento mais avançado para permitir sua implementação.

Assim, sabendo que a customização de um determinado projeto depende, em certa medida, das características do projeto original do apartamento, o passo inicial, apresentado no presente artigo, foi a realização de um estudo piloto desenvolvido com o objetivo de analisar em que medida as características formais e construtivas do projeto original (PO) influenciam na customização dos projetos reformados (PRs). É sabido, também, que muitos fatores podem influenciar na decisão de reformar ou não uma habitação, como a condição financeira, por exemplo. No entanto, essa fase da pesquisa se

3 Segundo Celani (2011), "um sistema generativo é um método indireto do projeto, no qual o projetista não se preocupa apenas com a solução de um problema em particular em um contexto específico. Ele (ou ela) procura criar um projeto mais ou menos genérico, que possibilite resolver problemas semelhantes em contextos diferentes". restringe apenas aos fatores de natureza formal e construtiva do projeto. É necessário, portanto, identificar as características da forma e da estrutura dos POs que pode permitir, restringir ou até mesmo impossibilitar as reformas.

Essa identificação é feita através do desenvolvimento: (1) de uma gramática descritiva e analítica de nove POs (Figura 1) de alto e médio padrão, construídos no Recife na primeira década do século XIX e que ressalta, dentre este corpus, os padrões de adjacência dos ambientes de cada apartamento; (2) de regras preliminares (feita com 12 PRs de dois POs) que farão parte de uma gramática de reforma, cujo objetivo, nesta fase, é evidenciar os padrões de reforma, como é descrito mais detalhadamente a seguir.
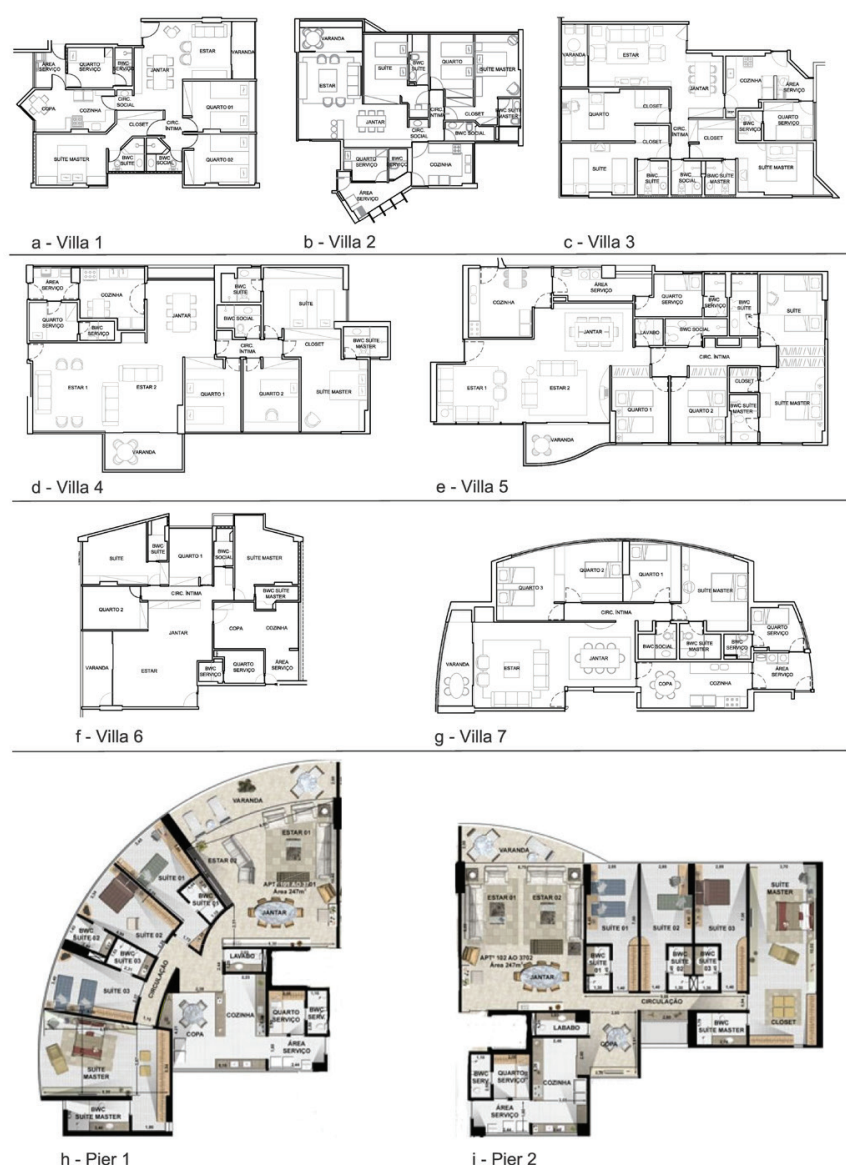
e - Villa 5

Figura 1: Projetos originais (POs).

\section{Sobre a gramática da forma}

A gramática da forma consiste em um sistema de geração de formas baseado em regras, e foi desenvolvido a partir das teorias da gramática generativa do linguista Noam Chomsky e do sistema de produção do matemático Emil Post, que consistia na substituição de caracteres em uma sequência de letras com o objetivo de geração de novas sequências. Criada por Stiny e Gips (1972), pode ser descrita como "um sistema de algoritmos para criar e entender 
projetos diretamente através de computação com formas [...] é uma série de regras que são aplicadas passo a passo a formas para gerar uma linguagem de projeto" (Eloy, 2012, p. 51).

Essa teoria vem sendo explorada, ao longo do tempo, em diversas aplicações para resolução de problemas projetuais, investigando como gerar projetos a partir de uma forma inicial, por meio de sucessivas aplicações de regras de forma (Duarte, 2007; Knight, 2000; Celani et al, 2006). Desde então, diversos tipos de gramáticas foram elaboradas e aplicadas à arquitetura. Dentre diversos trabalhos, podem ser citados os estudos desenvolvidos por Stiny e Mitchell (1978) nas Villas Palladianas, que objetivava definir o estilo neoclássico do arquiteto Andrea Palladio. Análises realizadas por Koning e Eizenberg (1981), sobre as casas da pradaria de Frank Lloyd Wright, buscando os princípios de composição definidos por ele. E o estudo de José Duarte (2005), que a partir de análises referentes ao conjunto habitacional da Malagueira, localizado em Évora, de Álvaro Siza, buscou a personalização de habitações em série. Duarte trabalha uma gramática que busca fornecer moradias de alta qualidade, porém com custos acessíveis, já que seria a criação de habitações em série, e, possivelmente, traria consigo um processo construtivo mais acelerado.

De uma maneira geral, as gramáticas da forma podem ser analíticas ou originais (Duarte, 2005). Elas são analíticas quando usadas como ferramenta de análise de um grupo de projetos - o corpus, descrevendo-os através de uma única linguagem que os represente. São originais quando é concebida para criar novos projetos, que atendam a requisitos pré-estabelecidos.

Neste caso, assim como a gramática proposta por Duarte (2005), a gramática da customização teria uma vertente analítica - uma vez que as regras são inferidas a partir de projetos já customizados pelos moradores, e uma original, já que a proposta final é que ela seja aplicada para novas customizações, com o mínimo de custos para o empreendedor e o cliente. Já a gramática para projetos de apartamentos potencialmente flexíveis é inteiramente original, devendo, para tanto, seguir todos os passos necessários para o seu desenvolvimento.

\section{Sobre o processo de desenvolvimento das gramáticas}

Tendo o formalismo da gramática da forma como base de análise, a presente investigação vem sendo desenvolvida em duas etapas:

Etapa 01: Criação de uma gramática analítica dos POs, que define regras graduadas de acordo com seus potenciais para reforma. O objetivo é descrever, através de regras que formem uma mesma linguagem, as características formais (de adjacência), e construtivas dos projetos originais (POs).

Etapa $02^{4}$. Criação de uma gramática analítica dos projetos reformados (PRs) - a gramática das reformas. O objetivo

4 Apresentada aqui através do estudo piloto com projetos reformados de apenas dois POs. desta primeira fase de desenvolvimento dessa gramática é identificar as regras de reformas mais importantes - os padrões de reforma (analisadas através dos PRs), pois a partir dessa identificação, será possível graduar as regras da gramática analítica dos POs de acordo com seus potenciais de reforma.

Para ilustrar o desenvolvimento das duas gramáticas, toma-se o projeto Villa 4 como exemplo (Figura 2). Como um dos requisitos primordiais quando se trata de processo de customizar um projeto é o da adjacência entre os ambientes (Eloy, 2012), a gramática dos POs se baseia neste principio e apresenta, também, informações relativas aos aspectos construtivos da edificação, que podem permitir, restringir ou impossibilitar as reformas. Assim, o primeiro passo é analisar as relações de adjacência entre espaços convexos funcionais ${ }^{5}$ e estas são representadas como as regras da gramática. É importante salientar que, neste nível de desenvolvimento, as regras abstraem a forma e dimensão real do espaço convexo', sendo estes representados por uma forma genérica - um polígono convexo, com a indicação de um marcador (label). O uso de marcadores em uma gramática da forma tem como objetivo reduzir a ordem de simetria de uma forma, restringindo a maneira como as regras podem ser aplicadas, permitindo também maior controle sobre os resultados. $\mathrm{Na}$ presente pesquisa, os marcadores também apresentam os aspectos funcionais dos ambientes.

Assim, tomando-se o Hall como ponto de partida (Figura 2), percebe-se que este é adjacente ao espaço convexo do Estar 1; o Estar 1, adjacente ao espaço convexo do Quarto e BWC de serviço e ao Estar 2, e assim por diante. Esses atributos de adjacências de cada espaço são transformados em regras preliminares que formam a gramatica dos POs. Após a análise dos nove POs que formam o corpus, a validação da gramática, assim como os ajustes necessários, é feita através da derivação de cada um dos POs (Figura 3).

5 Conforme comentado, essa investigação faz parte de uma pesquisa maior, onde também são analisados os aspectos configuracionais dos projetos, de acordo com os conceitos e métodos descritos na Teoria da Lógica Social do Espaço (Hillier \& Hanson, 1984). Para a análise desses aspectos, os ambientes do apartamento são representados através da menor quantidade dos maiores espaços convexos possíveis de se delimitar entre as barreiras físicas da edificação, que podem ser fixas (paredes, desníveis de piso, etc.) ou móveis (arranjo do mobiliário). Quando as barreiras físicas são os mobiliários, esse convexo é denominado de "funcional". Assim, o convexo funcional é adotado para que a representação do projeto nas análises configuracionais e formais sejam as mesmas.

6 Assim como a gramática da transformação, proposta por Eloy (2012), a gramática dos POs precisa ser paramétrica devido à variedade de formas e dimensões dos ambientes encontrados nos apartamentos. É apresentado o primeiro esboço da gramática, onde as regras preliminares são representadas através de polígonos convexos. No desenvolvimento da gramática, esses polígonos serão parametrizados, acrescentando informações relativas ao número de vértices e ao número e dimensão dos lados. 


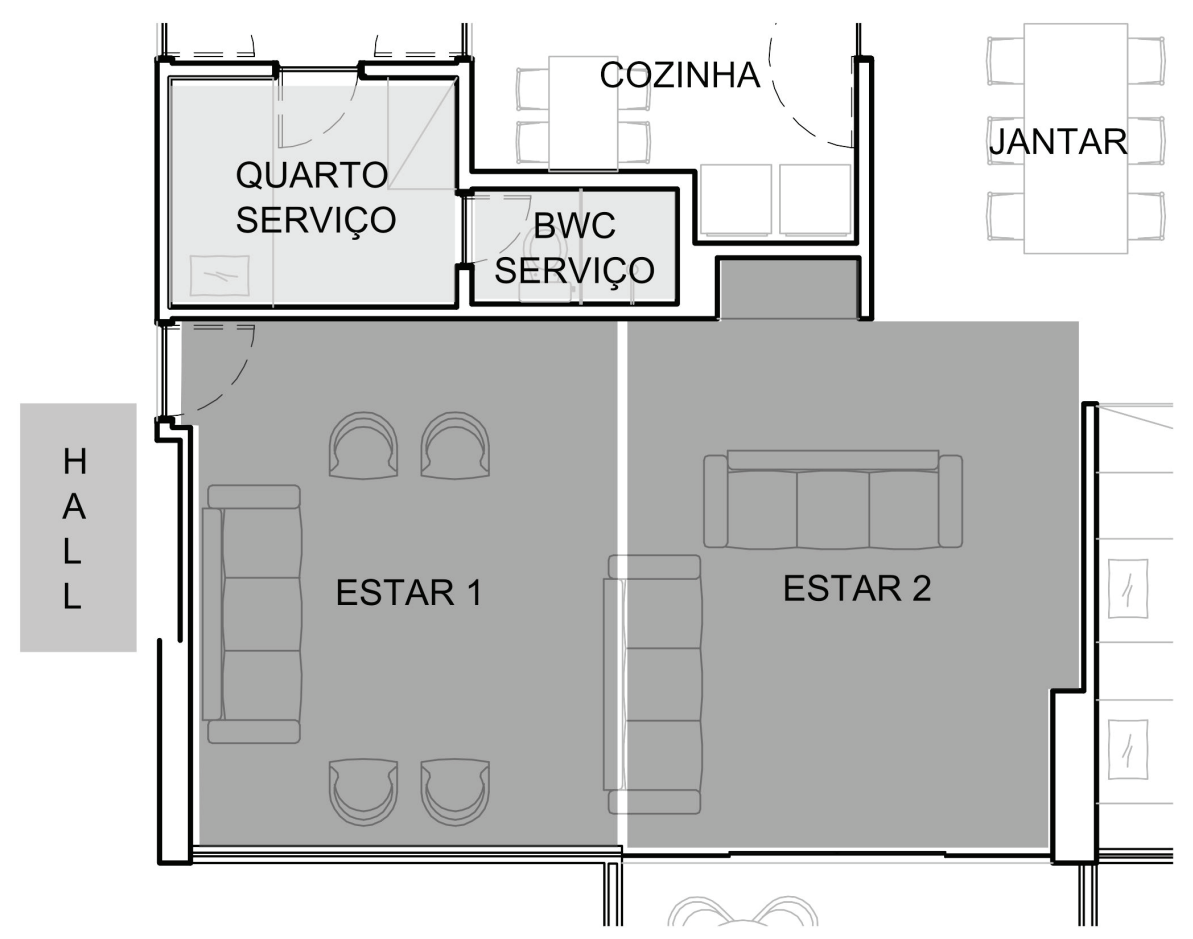

REGRAS

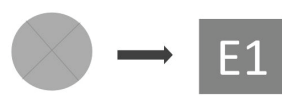

$\mathrm{E} 1 \rightarrow \mathrm{E} 1 \mathrm{E}^{2}$

E1 $\rightarrow$ E1 QS

E1 $\rightarrow$ E1 Bse

Figura 2: Processo para inferir as regras da gramática dos POs.

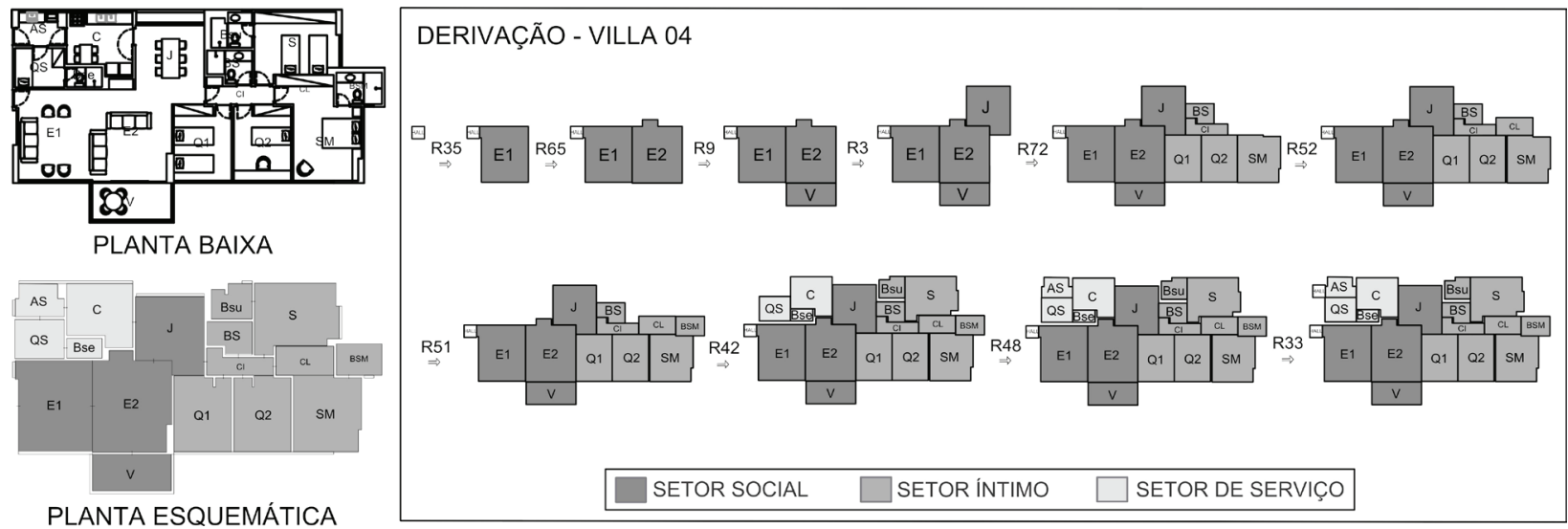

Figura 3: Exemplo de derivação de um dos POs.

Para facilitar o entendimento e a identificação de padrões de adjacência existentes entre os projetos, as regras da gramática foram descritas numa planilha (Tabela 1). Dessa maneira é possível comparar as informações dos nove POs que forma o corpus. A regra R28, por exemplo, que representa a relação de adjacência do Hall com o Estar1, é verificada em $50 \%$ dos POs os Villa 2, 3, 4 e 7, enquanto que a regra $\mathrm{R} 66$, que indica a relação de adjacência do Estar com o Quarto de Serviço, aparece apenas no Villa 5.

Além da adjacência, são também identificados os elementos estruturais que agem como restrições ao processo de customização. Além dos shafts e pilares, são identificadas as relações de adjacência que apresentam limites em comum menores que dois metros, pois estas podem dificultar a junção de determinados ambientes. Esses dados foram representados em planta (Figura 4) e em planilha (Tabela 1). A representação das restrições na planilha permite a fácil identificação dos elementos estruturais em cada uma das regras da gramática dos POs.

Como comentado, para graduar as regras dessa gramática de acordo com seus potenciais para reformar é preciso identificar os padrões de reforma - tarefa feita através do desenvolvimento das regras preliminares da gramatica da reforma. O processo para inferir tais regras é feito de maneira simplificada, também não parametrizada ${ }^{7}$, num estudo piloto que apresenta um corpus

7 A forma dos ambientes representados nas regras preliminares da gramática da reforma também é genérica, feita através de polígonos convexos, que, posteriormente, serão parametrizados. 


\begin{tabular}{|c|c|c|c|c|c|c|c|c|c|c|}
\hline Regra & Descrição & $v_{1}$ & $\mathbf{v} 2$ & $v_{3}$ & 104 & v5 & v6 & v7 & & $\mid \mathrm{P} 2$ \\
\hline R1 & hall social adjacente ao jantar & 0 & & & & 0 & 0 & & 0 & 0 \\
\hline R2 & jantar adjacente ao estar & 2 & 2 & 2 & 2 & 2 & 2 & 2 & 2 & 2 \\
\hline R3 & estar adjacente ao jantar & & 2 & 䒚茥 & 2 & & & 2 & & \\
\hline R9 & estar adjacente ao quarto & 2 & & |lin| & $\|\mid\|$ & 2 & & & & \\
\hline R20 & suite sec.adjacente ao BWC suite & 1 & fi & 1 & ti & 1 & 聮 & t: & & $\overline{E=}=$ \\
\hline R24 & copa adjacente à área de serviço & 罦铻 & & & & & & & & \\
\hline R28 & hall social adjacente ao estar & & 0 & 0 & 0 & & & 0 & & \\
\hline R31 & jantar adjacente ao quarto de serviço & & 0 & & & 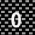 & 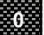 & & & \\
\hline R35 & cozinha adjacente à área de serviço & & 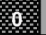 & 1 & $\theta=$ & 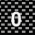 & 1 & 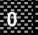 & 箂 & 淁睃 \\
\hline R60 & suite master adjacente à suite sec. & & & & & 2 & & & 2 & 2 \\
\hline R61 & jantar adjacente à copa & & & & & & 1 & 篰旁 & & \\
\hline R66 & copa adjacente ao quarto de serviço & & & & & & 0 & & & \\
\hline R84 & suite sec. adjacente a outra suite sec. & & & & & & & & & $\|$ | \\
\hline
\end{tabular}

Tabela 1: Parte das regras que formam a gramática dos POs - descrições, POs onde são verificadas e indicação de existência de alguma restrição construtiva.

de 12 PRs, originados dos Villa 4 e $5^{8}$.

Semelhante à gramática proposta por Eloy (2012), a gramática da reforma não apresenta uma forma inicial, já que esta corresponde aos diferentes projetos originais. Por outro lado, o processo de inferir regras se diferencia na medida em que as regras são inferidas através de projetos reformados já existentes, enquanto na gramatica da transformação (ELOY, 2012), esse processo é feito através de possíveis projetos propostos por diferentes arquitetos.

O processo para inferir regras tem como objetivo capturar a lógica subjacente ao projeto, ou seja, descrever em regras os padrões de reforma reconhecidos nos projetos. Tendo isso como pressuposto, na análise do corpus de PRs, percebe-se que as customizações são feitas usando mudanças espaciais semelhantes, em espaços com funções, forma e dimensão diferentes. Essas mudanças espaciais foram classificadas em três grupos de regras formais:

a. Regras formais de aglutinação (RFA): quando dois espaços convexos adjacentes são aglutinados inteiramente e se transformam em um só;

b. Regras formais de divisão (RFD): quando um espaço convexo é dividido em outros dois espaços;

c. Regras formais de alteração escalar (RFE): quando dois espaços convexos adjacentes alteram suas dimensões - um ficando maior que o outro.

8 Estes POs foram selecionados para o estudo piloto por apresentarem dimensão e programa arquitetônico semelhantes.
Soma-se a essas regras formais outro tipo de mudança muito frequente nos PRs - a alteração de rótulo, onde um espaço convexo permanece com a mesma forma e dimensão, alterando apenas sua função. Esse tipo de mudança gera as regras da gramática denominadas Regras de alteração de rótulo (RAR), completando, assim, os quatro grupos de regras da gramática da reforma (Figura 5).

Como esses quatro grupos de regras podem ser referentes a espaços com forma, função e dimensão distintos, sempre que se atribui o marcador ao espaço convexo, a regra passa a se chamar Regra formal de adição 1 (RFA1), ou Regra formal de divisão 2 (RFD2), e assim por diante (Figura 6). Dessa maneira, assim como Eloy (2012, p. 188),

...em vez de ter diferentes regras de mudança de forma para cada uma dasáreas funcionais, era preferível ter um grupo regras de mudança de forma apenas com a forma igual e as condições serem diferentes de acordo com as restrições funcionais...

Apesar de cada grupo de PRs apresentarem uma gramática distinta - a gramática do Villa 4 e a gramática do Villa 5, as regras das gramáticas dos dois grupos recebem o mesmo nome, também descritos em planilha (Tabela 2), para poder identificar os padrões de reforma mais utilizados e assim, poder graduar as regras da gramática dos POs, de acordo com seus potencias para reformar.

Os padrões de reforma são referentes às regras da gramática da reforma que aparecem em, no mínimo, 50\% dos projetos reformados ou que estão presentes nas gramáticas do Villa 4 e Villa 5 ao mesmo tempo. No entanto, algumas outras regras que aparecem em, pelo menos, 30\% dos PRs, também são levadas em consideração para a graduação das regras da gramática dos $\mathrm{PO}$, que é feita da seguinte maneira:

Potencial para reformar alto (pontuação 2): atribuída às regras dos POs que apresentam a adjacência indicada na representação da direita dos padrões de reforma fortes;

Potencial para reformar médio (pontuação 1): atribuída às regras dos POs que apresentam a adjacência indicada na representação da direita dos padrões de reforma fracos;

Potencial para reformar baixo (pontuação 0): atribuída às regras dos $\mathrm{POs}$ que apresentam adjacência que não foram consideradas relevantes nas reformas analisadas;

Por fim, esses valores do potencial para reformar são transcritos na Tabela 1. Nas regras onde era verificada alguma das restrições construtivas mencionadas anteriormente, o potencial para reformar diminui. A regra R9, por exemplo, é uma das que apresentam um forte potencial para reformar. (valor 2) No entanto, nos Villa 3 e Villa 4 é verificado um pilar entre a sala de estar e o quarto, o que restringe o potencial para médio (valor 1), nesses dois POs. 


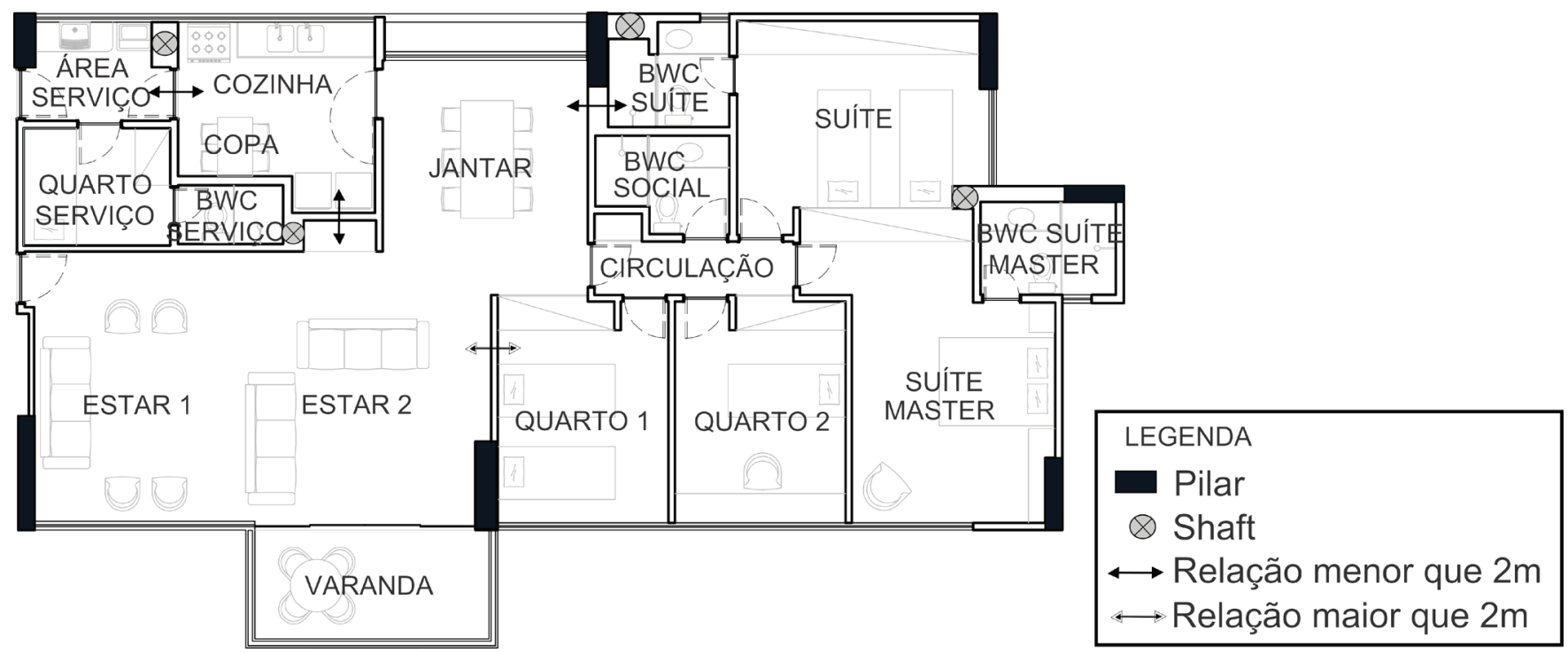

Figura 4: Indicação das restrições construtivas em planta.

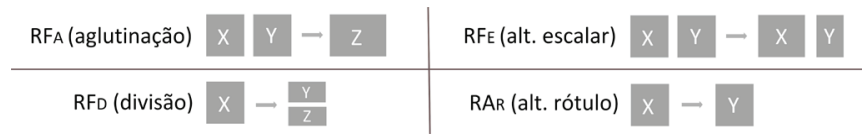

Figura 5: Grupos de regras que constituem a gramática da reforma.

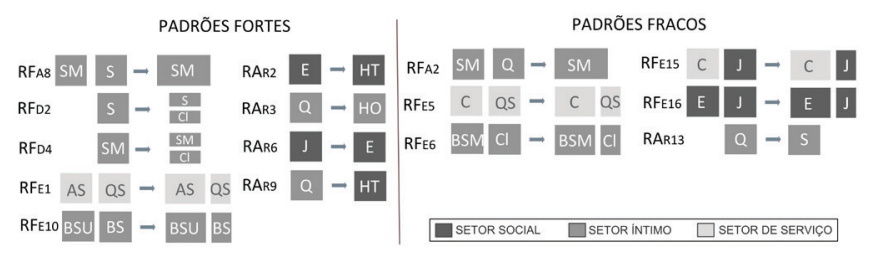

Figura 6: Regras da gramática da reforma identificadas como os padrões de reforma.

\section{Sobre a gramática dos POs e a gramática da reforma}

A aplicação dos princípios teóricos e metodológicos da gramática da forma na análise dos projetos de apartamento originais e reformados explicita certos padrões da sua organização formal - os padrões de adjacência nos POs e os padrões de reforma nos PRs.

A gramática dos POs descreve a organização formal dos projetos originais dos apartamentos, levando em conta a adjacência entre os ambientes. Dentre as 84 regras que formam a gramática, 24 podem ser consideradas padrões de adjacência, que aparecem em aproximadamente $50 \%$ dos POs. Esse resultado confirma a padronização característica desse tipo de projeto, mostrando que existe uma linguagem formal onde a adjacência de certos ambientes é aplicada sistematicamente, seja por razões funcionais (e, praticamente, impositivas), seja em função da forma geral do projeto, seja por razões especulativas (que já antecipam a possibilidade de reformar).

Exemplos de adjacência por questões funcionais incluem basicamente ambientes onde existe a acessibilidade direta entre eles, como um dormitório e seu banheiro privativo (R20), a sala de jantar e a de estar (R2 e R3), a cozinha e a área de serviço (R24), por exemplo. Vale destacar que todas as regras desse tipo (adjacência por questões funcionais) também fazem parte daquelas identificadas como padrão de referencia.

Já as adjacências resultantes da forma geral do projeto podem acontecer entre ambientes que não requerem tal atributo formal, como é o caso da Regra R31, entra a sala de jantar e o quarto de serviço, verificada em três POs (Villas 2, 5 e 6). A adjacência aqui referida como especulativa foi classificada como tal após a identificação dos padrões de reforma (vistos a seguir). É verificada principalmente entre: um dos dormitórios e os ambientes sociais do apartamento (encontrada em todos os $\mathrm{POs}^{9}$ - R9 e R61) e entre a suíte principal e um dormitório ou uma suíte secundária (R34 e R60).

Antes de falar na graduação das regras dessa gramática, são analisadas as regras de reforma mais frequente, dentre as quais estão os padrões de reforma. Nos PRs, mesmo que seja buscada uma singularidade do ambiente doméstico através das mais variadas soluções projetuais (Griz, 2012), percebe-se que esta "variedade" apresenta certa uniformidade, verificada através da aplicação de estratégias compositivas semelhantes. A gramática dos PRs está justamente explicitando essa uniformidade, mostrada pela aplicação sistemática da certas regras de reforma para projetos distintos, formando, assim, os padrões de reforma.

Esses padrões também são classificados como fortes (regras de reforma encontradas nas gramáticas do Villa 4 e Villa 5 ao mesmo tempo) ou fracos (regras de reforma encontradas em pelo menos 50\% dos PRs em uma das duas gramáticas desenvolvidas). Apenas nove regras de reforma (10\% do total) foram consideradas fortes (Tabela 2 e Figura 6): RFA8, RFD2; RFD4, RFE1, RFE10, RAR2, RAR3, RAR6, RAR9. Já as regras fracas na gramática do Villa 4 e no Villa 5 são três em cada uma: RFA2, RFE5, RFE6, no Villa 04 e RFE15, RFE16 e RAR13 no Villa 5.

9 Vale salientar que essa adjacência só é possível pelo formato geral do projeto, que permite que os setores social e íntimo sejam também adjacentes. 


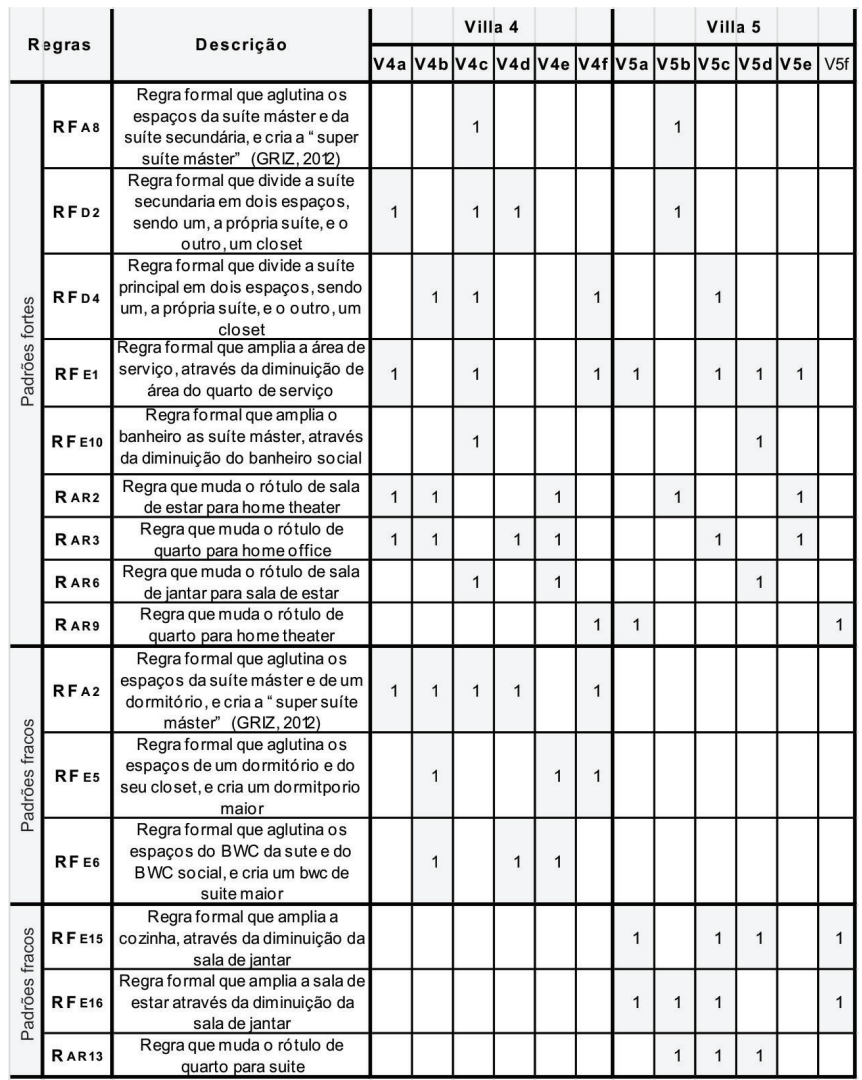

Tabela 2: Padrões de reforma - regras, descrição e PRs onde são verificados.

Essa uniformidade resultante do processo de customização que geram os PRs, principalmente através da aplicação sistemática dos padrões de reforma, reforça duas constatações verificadas em trabalhos anteriores (Griz, 2012): (1) a valorização dos ambientes do setor social e da suíte principal; (2) a desvalorização do quarto de serviço.

Um exemplo da valorização dos ambientes do setor social é verificado com a aplicação da regra RAR9 (Figura 7), que só é possível caso o projeto seja formado com a regra R9, da gramática dos POs. Ou seja, só acontece quando a sala de estar é adjacente a um dos dormitórios e a área desse último é incorporada para o setor social para inserir atividades especializadas, como o home theater.
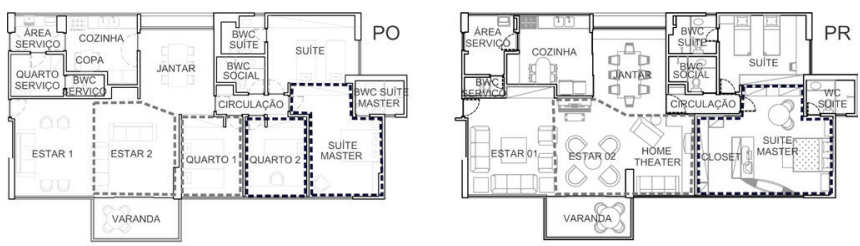

REGRAS FORTES

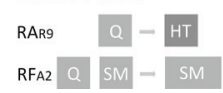

Figura 7: Exemplo de projeto onde os dois principais padrões de reforma são verificados.

Regras semelhantes são utilizadas para valorizar a suíte principal. As regras RFA2 e RFA8 ilustram esse fato. Na primeira, a suíte principal é adjacente a um dos quartos (Figura 7); na segunda, a uma suíte secundária. Em ambas as regras, a suíte principal é aumentada devido à incorporação da área do ambiente adjacente a ela. Vale ressaltar que, da mesma maneira que o exemplo anterior, essa valorização só é possível quando da existência das regras R34 e R60 na gramática dos POs.

Por fim, tendo identificado os padrões de reforma (e os ambientes que formam as regras que os compõe), é possível graduar as regras da gramática dos $\mathrm{POs}$, de acordo com seus potenciais para reformar: fraco (0), médio (1) e forte (2). A média dos potenciais de reformas das regras utilizadas para a derivação de cada um dos POs indica seu potencial geral para reformar (Tabela 3).

Segundo essa lógica, o Villa 7, e, com um valor bem próximo a ele, o Villa 5, são os POs que apresentam os maiores potenciais para serem reformados. Ou seja, caso as famílias que adquiriram esses apartamentos optem por reforma-los, elas terão um reduzido número de restrições e uma maior flexibilidade no processo de customização, em se comparando com os demais POs. No extremo oposto está o Villa 3, sendo o $\mathrm{PO}$ com menor potencial para reformar.

\begin{tabular}{|c|c|c|c|}
\hline \multicolumn{2}{|c|}{ GAP } & \multicolumn{2}{c|}{ Pot ref } \\
\hline 3,25 & Pier 01 & Villa 07 & 0,88 \\
\hline 3 & Villa 01 & Villa 05 & 0,85 \\
\hline 3 & Villa 02 & Villa 04 & 0,74 \\
\hline 2,83 & Villa 04 & Pier 02 & 0,74 \\
\hline 2,67 & Villa 05 & Pier 01 & 0,69 \\
\hline 2,5 & Villa 03 & Villa 06 & 0,65 \\
\hline 2,5 & Villa 06 & Villa 02 & 0,64 \\
\hline 2 & Pier 02 & Villa 01 & 0,62 \\
\hline 1,17 & Villa 07 & Villa 03 & 0,55 \\
\hline
\end{tabular}

Tabela 3: Potencial para reformar X GAP em cada PO.

Cruzando esses dados com os verificados para o Grau de Alteração do Projeto - GAP (Griz, 2012), é possível ver que o Villa 7, por exemplo, apesar de apresentar o maior potencial para reformar, seus PRs foram os menos reformados. Os demais POs não apresentaram nenhum relação relevante entre o potencial para reformar e o GAP.

Sabe-se que as razões pelas quais um projeto é ou não reformado pelo cliente podem ser variadas - desde fatores econômicos a fatores de ordem pessoal. Essas duas últimas poderiam ser descritas como razoes "abstratas" que exercem influencia no quanto um projeto de apartamento pode ser customizado. Dentro desse raciocínio, as razões "concretas" seriam os atributos formais e construtivos do projeto, podendo 
ser consideradas como fundamentais, pois mesmo que os fatores econômicos e pessoais sejam favoráveis à reforma, se a forma (vista aqui, através da adjacência entre ambientes) e os elementos construtivos não facilitarem, a customização pode ser fraca ou até inexistente.

Sabendo disso, e diante dos resultados expostos, podese dizer que a forma dos projetos analisados apresentou pouca influencia no grau de alteração dos projetos reformados, indicando, assim, que fatores de outra natureza foram mais determinantes no processo de customização dos PRs analisados.

Por fim, valedestacar um resultado curioso(a ser explorado em análises com um corpus de PRs maior). Os quatro POs que apresentam um maior potencial para reformar - em ordem decrescente, Villas 7, 5, 4 e P1, apresentam uma forma geral do projeto que tendem para um quadrado. Contrariamente, os cinco POs cujos potenciais para reformar foram baixos, a forma geral do projeto tende a ser mais retangular. Seria esse um dado relevante na medição da influencia da forma na customização de projetos de apartamentos?

\section{Reformar ou não reformar?}

Reformar ou não reformar? Eis a questão! A dúvida cabe e é bem pertinente no contexto do atual mercado imobiliário de projeto de apartamentos. Produto caracterizado pela padronização de soluções, o projeto de apartamentos originalmente oferecido pelos empreendedores nem sempre se adequa às demandas dos compradores, fazendo com que o este opte por customiza-lo, sempre que possível, de acordo com seus desejos e necessidades.

Por essa razão, a investigação procurou saber se o grau de alteração (o quanto um projeto original foi reformado) dos projetos reformados sofre influencia das características formais e construtivas do projeto que lhes deu origem (PO). Os resultados, embora ainda parciais, mostram que essa questão não é definida analisando-se apenas esses aspectos formais e construtivos do projeto, uma vez que a influencia deles no processo de reforma foi fraco.

No entanto, os aspectos mais importantes e relevantes dessa fase da instigação para a pesquisa como um todo foi a aplicação do formalismo da gramática da forma como método de análise. O desenvolvimento das gramáticas, ainda que em nível preliminar, teve um propósito que foi atendido (medir a influencia da forma na customização de apartamentos). Em se tratando dos objetivos gerais da pesquisa, essas gramáticas precisam ser mais bem desenvolvidas10 para que seja possível tanto criar projetos originais potencialmente customizáveis (através da gramática dos POs), quanto para proporcionar a customização em massa (gramatica dos PRs).

\section{Agradecimentos}

Os autores agradecem a Fundação de Amparo à Ciência e Tecnologia do Estado de Pernambuco (FACEPE), pelo apoio financeiro a essa pesquisa (através do APQ - 0495 - 6.04 / 14), e às bolsistas de iniciação científica Maria Augusta Rodrigues de Holanda e Thais Castelo Branco, pela ajuda na finalização do estudo piloto.

\section{Referências}

Brandão, D. (2002). Diversidade e potencial de flexibilidade de arranjos espaciais de apartamentos: Uma análise do produto imobiliário no Brasil. (Tese de Doutorado em Engenharia de Produção). Universidade Federal de Santa Catarina, Florianópolis.

Celani, C. (2011). Algorithmic Sustainable Design. Vitruvius, v.116. Disponível em: http://www.vitruvius.com.br/revistas/ $\mathrm{read} / \mathrm{resenhasonline/10.116/3995}$

Celani, G.; Cypriano, D.; Godoi, G.; Vaz, C. E. V. (2006) A gramática da forma como metodologia de análise e síntese em arquitetura. Conexão - comunicação e cultura/Universidade de Caxias do Sul, Caxias do Sul, v. 5, n. 10, p. 180-197..

Duarte, J. P. (2005). Towards the mass customization of housing: the grammar of Siza's houses at Malagueira. Environment and Planning B: Planning and Design, v. 32, p. 347-380.

Duarte, J. P. (2007). Personalizar a habitação em série: Uma Gramática Discursiva para as Casas da Malagueira do Siza. Lisboa: Ed. Fundação Calouste Gulbenkian.

Eloy, S. (2012) A transformation grammar-based methodology for housing rehabilitation: meeting contemporary functional and ICT requirements. (PhD). Universidade Técnica de Lisboa, Lisboa.

Fischer, T.; Herr, C. M. (2001). Teaching Generative Design. In 4th International Generative Art Conference, Generative Design. Milão. Disponível em: <http://www.generativeart. com>. Acesso em: 14 abril 2015.

França, F. C. (2008). Indisciplina que muda a arquitetura: a dinâmica do espaço doméstico no Distrito Federal (Tese de Doutorado em Arquitetura e Urbanismo). Universidade de Brasília, Brasilia.

Griz, C. (2012). Quando o luxo é necessário. Sobre projetos de apartamentos no Recife. (Tese de Doutorado em Desenvolvimento Urbano). Universidade Federal de Pernambuco, Recife.

Griz, C. (2014) Projetos de apartamentos do Recife: relação entre morfologia e modos de morar contemporâneos (Projeto de pesquisa). Universidade Federal de Pernambuco, Recife.

Griz, C.; Amorim, L.; Loureiro, C. (2014). Entre a oferta e a demanda: a elite em busca do "morar bem", In XV Encontro Nacional De Tecnologia Do Ambiente Construído. Maceió.

Hanson, J. (1998). Decoding homes and houses. Cambridge: Cambridge University Press.

Hillier, B.; Hanson, J. (1984). The social logic of space. Cambridge: Cambridge University Press.

Knight, T. (2000). Shape Grammars in education and practice: history and prospects. International Journal of Design Computing, v. 2. Disponível em < http://www.mit. edu/ tknight/IJDC/ >. Acesso em: 24 set. 2015.

Kolarevic, B. (2001). Desining and Manifacturing Architecture in the digital age. In Architectural Information Management - 05 Design Process (3), p. 117-123.

Koning, H.; Eizenberg, J. (1981). The language of the prairie: Frank Lloyd Wright's prairie houses. In: Environment and Planning B: Planning and Design, v.8, p.295-323.

Mendes, L.T. (2014). Personalização de Habitação de Interesse Social no Brasil: o caso da implantação urbana em conjuntos habitacionais. (Tese de Doutorado em Arquitetura) Universidade Estadual de Campinas, Campinas. 
Mussi, A. Q. (2011). Projeto de HIS Evolutiva: Gramática da Forma para Ampliação da Unidade Habitacional Mínima. In 2o Simpósio Brasileiro de Qualidade do Projeto no Ambiente Construído X Workshop Brasileiro de Gestão do Processo de Projeto na Construção de Edifícios. Rio de Janeiro.

Paula, V. D. C. (2007). Espaço e sociedade: Apartamentos no Rio de Janeiro no século XX (Tese de Doutorado em Arquitetura e Urbanismo). Universidade Federal do Rio de Janeiro.

Stiny, G.; Gips, J. (1972). Shape grammars and the generative specification of painting and sculpture In 7th Ifip Congress.
Amsterdam. Disponível em: <http://www.shapegrammar. org/ifip/ifip1.html>. Acesso em: 05 nov. 2014.

Stiny, G., Mitchell, W. J. (1978). The Palladian Grammar. In: Environment and Planning B: Planning and Design 5 5-18.

Tramontano, M. (2004). Apartamentos e vida privada na cidade de São Paulo (Tese de Livre Docência). Universidade de São Paulo, São Carlos.

Trigueiro, E. (1994). Change in domestic space design (Tese PhD em Advanced Studies). University College of London. 